\title{
A step toward facilitating ethics review of human research in Australia
}

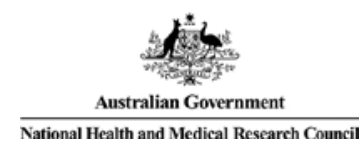

One of the main obstacles for Australian researchers applying for ethics approval of their research has been the absence of an application form that can deal equally well with all research and, more importantly, simplify the application and ethics review process. Such a form would enable the consideration of the critical ethical issues related to a proposed research project by both the applicant and the reviewing committee. A fresh perspective on the ethics application process has been requested both by applicants and ethics committee members.

The National Health and Medical Research Council (NHMRC) has sought to respond to this need through the development of a concise, streamlined application form, supported by a contemporary technology platform, to facilitate efficient and effective ethics review for all research involving humans. The development of this form - the Human Research Ethics Application (HREA) - is part of a suite of initiatives funded through government budget measures to streamline ethics approval processes.

Designed with a new structure and logic, and with sophisticated filters that generate questions specific to each application, the HREA will assist researchers to consider how the ethical principles and standards that apply to their research will be addressed. We anticipate that the new form will be welcomed by researchers and reviewers alike, and will lead to more efficient human research ethics review and approval.

The continuing engagement of the research community and the ethics committees that support the research is integral to the development of this application. The NHMRC will soon be seeking feedback on a preliminary version of the HREA (https://www.nhmrc.gov.au). An initial launch of the HREA is planned for early 2016.

Gordon McGurk Joel Ceramidas Jeremy Kenner National Health and Medical Research Council, Canberra, ACT. gordon.mcgurk@nhmrc.gov.au doi: 10.5694/mjal5.01149 